\title{
RELATO DE EXPERIÊNCIA DE DOUTORANDOS EM AULAS REMOTAS EMERGENCIAIS
}

\section{EXPERIENCE REPORT OF DOCTORAL STUDENTS IN EMERGENCY REMOTE CLASSES}

\author{
Lucas dos Santos, Sérgio Donha Yarid, Alba Benemérita Alves Vilela, Evely Rocha Lima, \\ Rhaine Borges Santos Pedreira, Thalane Souza Santos Silva
}

Universidade Estadual do Sudoeste da Bahia

\section{Abstract}

This study aimed to describe the experience of doctoral students in the health field in the face of the subjects taken remotely due to the pandemic of COVID-19. The development of the experience revealed that the emergence of the pandemic generated frustration in the expectations of the doctoral students, since the face-to-face teaching modality provides important academic and personal experiences. In addition, problems with internet connection, damage to physical and mental health and unfavorable ergonomic conditions were reported by the students. However, the commitment of the postgraduate program, of teachers and students, to satisfactorily conduct the teaching-learning process in the remote remote teaching modality was perceived. Potentialities were also verified, such as the reduction in expenses with transportation, accommodation and meals for students residing in other cities, as well as the possibility of participating virtually in scientific events held in other locations. This signals the potential of remote education to increase access to postgraduate programs for those residing in less favored regions. It is concluded that the use of the remote education modality was positive, although challenging for all involved, especially with regard to quality internet access and the lack of face-to-face communication.

Keywords: Online Education; Health Postgraduate Programs; Pandemics; COVID19.
Resumo

Este estudo teve como objetivo descrever a experiência de discentes de doutorado na área da saúde diante das disciplinas cursadas de forma remota em decorrência da pandemia de COVID19. O desenvolvimento da experiência revelou que o surgimento da pandemia gerou frustração nas expectativas dos doutorandos, uma vez que a modalidade de ensino presencial proporciona importantes experiências acadêmicas e pessoais. Ademais, problemas de conexão com a internet, prejuízos na saúde física e mental e condições de ergonomia desfavoráveis foram relatados pelos discentes. Contudo, percebeu-se o empenho do programa de pós-graduação, dos docentes $e$ discentes em conduzir o processo de ensinoaprendizagem na modalidade de ensino remoto emergencial de forma satisfatória. Potencialidades foram também verificadas, como a redução dos gastos com transporte, hospedagem e alimentação dos discentes que residem em outras cidades, bem como a possibilidade de participar virtualmente de eventos científicos realizados em outras localidades. Isso sinaliza o potencial do ensino remoto em aumentar o acesso aos programas de pós-graduação para aqueles que residem em regiões menos favorecidas. Conclui-se que o emprego da modalidade de ensino remoto foi positivo, apesar de desafiador para todos os envolvidos, em especial no que se refere ao acesso à internet de qualidade e a falta de comunicação presencial.

Palavras-chave: Educação Online; Programas de Pós-Graduação em Saúde; Pandemias; COVID-19. 


\section{Introdução}

No início do ano de 2020 a Organização Mundial da Saúde (OMS) declarou uma emergência de saúde pública de nível internacional, dada à alta transmissibilidade e capacidade de disseminação geográfica da COVID-19, doença causada pelo novo Coronavírus (Sars-Cov-2), caracterizando-a como pandemia em março do mesmo ano. ${ }^{1}$

Diante desse contexto inesperado, os países afetados iniciaram a implantação de medidas sanitárias como o distanciamento social, o incentivo à higienização das mãos, uso de máscaras de proteção, adoção de etiquetas respiratórias e suspensão da oferta de serviços não essenciais, no intuito de conter a disseminação da doença e minimizar o risco de esgotamento dos serviços de saúde. ${ }^{2}$

Tornou-se inevitável, portanto, a interrupção das atividades em instituições de ensino de todos os níveis, desde a educação básica até a pós-graduação, o que levou o Ministério da Educação e Cultura (MEC) a recomendar na portaria $\mathrm{N}^{\circ} 343$ de 17 de março de 2020 aulas mediadas por tecnologias digitais, como uma estratégia para substituir os encontros presenciais, minimizando os prejuízos na esfera educacional decorrentes da pandemia. ${ }^{3}$

Dadas às condições adversas da pandemia de COVID-19 para continuidade das aulas presenciais, diversas instituições de ensino públicas e privadas, aderiram às aulas remotas, que acontecem virtualmente de forma síncrona, o que permite contato simultâneo entre professores e alunos, e, consequentemente, o diálogo e discussão dos assuntos trabalhados. ${ }^{4}$

Cabe analisar que, embora essa alternativa de ensino tenha permitido a continuidade das aulas em diversas instituições, esse cenário trouxe à tona as fragilidades do sistema educacional. Tal fato exigiu esforços de todos os envolvidos no processo ensinoaprendizagem para adaptação a esta nova metodologia, além da necessidade de se repensar a equidade na educação diante da demanda de tornar as aulas virtuais acessíveis aos professores e alunos. Tornou-se urgente, portanto, repensar a vida em sociedade, mas também estabelecer estratégias capazes de minimizar os impactos socioeconômicos evidenciados pela COVID-19 ou decorrentes dela. ${ }^{5}$

Desta forma, este estudo tem como objetivo descrever a experiência de discentes de doutorado de um programa de pós-graduação (PPG) na área da saúde diante das disciplinas cursadas de forma remota em decorrência da pandemia de COVID-19.

\section{Metodologia}

Trata-se de um estudo descritivo, com abordagem qualitativa e do tipo relato de experiência, realizado por discentes de doutorado de um PPG na área da saúde, que vivenciaram $o$ ensino remoto durante a pandemia da COVID-19. Por se tratar de um relato de experiência, o presente estudo dispensa apreciação por um Comitê de Ética em Pesquisa.

Este relato de experiência foi elaborado como trabalho de conclusão de uma disciplina, proposto e orientado por seus docentes, sendo um Enfermeiro e um Cirurgião Dentista, ambos com doutorado na área da saúde. Além disso, compuseram também o cenário deste relato, quatro doutorandos do PPG, um Profissional de Educação Física, um Farmacêutico, um Fisioterapeuta e um Nutricionista.

O PPG em questão tem abordagem interdisciplinar que forma mestres e doutores em Ciências da Saúde, sediado em uma instituição pública de ensino superior no estado da Bahia. As aulas das disciplinas ofertadas pelo mesmo ocorrem tradicionalmente de forma presencial e regular, em dias e horários pré-estabelecidos pelo colegiado e demais professores do programa, e disponibilizados aos discentes anteriormente ao ato da matrícula. Apesar disso, diante da pandemia de COVID-19, e em virtude da necessidade de mitigar a disseminação da doença, as aulas presencias tornaram-se inviáveis e o programa aderiu ao ensino remoto.

O presente relato contempla a experiência vivenciada pelos discentes de doutorado durante 3 meses, ao participarem das aulas das disciplinas correspondentes ao primeiro período letivo do ano de 2020. Para realização das aulas remotas a equipe docente do PPG optou pela utilização da plataforma digital Google Meet, sendo as aulas ministradas nos mesmos dias e horários previstos no momento da matrícula dos alunos, e pelos mesmos professores responsáveis pelas disciplinas na modalidade presencial.

\section{Resultados e Discussão}

Os discentes aprovados na seleção para o doutorado na área de saúde, no final do ano 2019 , se matricularam nas disciplinas ofertadas e aguardavam o início das aulas no mês de março de 2020. As expectativas para o início das 
atividades acadêmicas da pós-graduação eram elevadas e estavam associadas a um novo desafio, aquisição de novos conhecimentos e experiências acadêmicas de importância para um estudante de pós-graduação.

Com o início da epidemia da COVID-19, a divulgação de notícias sobre sua gravidade pelas mídias sociais e jornais, e a iminência da suspensão dos transportes intermunicipais, culminou no cancelamento da aula de abertura do doutorado. A notícia foi recebida com alívio, pela possibilidade de se precaver do contágio, mas também com preocupação em relação a se, quando e como as aulas ocorreriam.

Desta forma, a possibilidade de o curso de pós-graduação ser iniciado em formato online gerou um sentimento de frustração e insegurança diante da nova realidade, marcada pela necessidade de organização de um ambiente e rotina que fossem favoráveis para o processo de ensino-aprendizagem.

Além disso, outra preocupação foi que o ambiente online, talvez, não fosse propício para uma boa interação e criação de laços entre os colegas de turma, tendo em vista que, algumas atividades da pós-graduação são realizadas em grupo. Neste sentido, a possibilidade de a falta de convivência resultar em implicações nas construções das atividades em grupo, gerou um receio por parte dos estudantes.

Paralelo a isso, a doença continuou se espalhando por todo o mundo e em março de 2020, a OMS declarou a COVID-19 como uma pandemia, já que neste momento mais de 118.000 casos da doença tinham sido notificados, espalhados por 114 países, com mais de 4.291 óbitos. ${ }^{6}$

No Brasil, os transportes já estavam suspensos, instituições de ensino fechadas, restrições de abertura do comércio e algumas cidades decretaram lockdown pela gravidade da situação. Era fato! A COVID-19 havia atingido todo o mundo! Números de casos e mortes aumentavam rapidamente, as notícias sobre lotação de hospitais, falta de leitos e respiradores e a morte de pessoas próximas assustavam a todos.

Desse modo, o retorno das aulas presenciais em unidades de ensino não era seguro, e nem uma prioridade. Foi quando em março de 2020 o MEC decretou a substituição das aulas presenciais por aulas em meios digitais. À medida que os estudos sobre a doença avançavam e, com os esforços das organizações e instituições internacionais e nacionais na tentativa de compreender e conter o avanço da pandemia, em junho de 2020, o MEC publicou a portaria № 544/202 autorizando a realização de aulas remotas em todos os níveis de ensino, incluindo o superior até 31 de dezembro de 2020. ${ }^{7}$ A recomendação do MEC foi acatada pelo Conselho de Ensino e Pesquisa da instituição de ensino superior em questão por meio das resoluções № 22/2020 e № 23/2020, que instituíram o ensino remoto emergencial na graduação e pós-graduação, respectivamente. , $^{89}$

No que diz respeito à adesão ao ensino remoto por parte do PPG, deve-se destacar a preocupação dos coordenadores, docentes e representantes discentes em dialogar e compreender a situação dos alunos e professores diante da alternativa de realizar as atividades de modo virtual. Inicialmente, uma sondagem foi realizada com o objetivo de avaliar as possibilidades de acesso à internet e às ferramentas como computadores ou smartphones, e qualidade de conexão à disposição dos mesmos.

Além disso, foram realizadas reuniões entre o corpo docente e discente tendo como pauta o ensino remoto, suas vantagens e desvantagens, dentre outros aspectos. A instituição promoveu iniciativas com o intuito de capacitar o corpo docente, instrumentalizando-o para ministrar as aulas virtualmente $\mathrm{e}$ desenvolver as atividades de ensino remoto na modalidade de aulas síncronas com uso da plataforma Google Meet.

Os professores e a turma demonstravam bastante empenho para que a experiência fosse exitosa, apesar de existir um receio de que as aulas apresentassem qualidade abaixo do esperado, que houvesse baixa adesão/participação dos alunos nas atividades, e especialmente, que problemas com a conexão de internet inviabilizasse ou atrapalhasse a ocorrência das aulas.

Considerando questões de ordem técnica, ocorreram situações em que parte das aulas foi perdida por instabilidades na conexão com a internet, bem como momentos de constrangimento, a exemplo de perder a conexão durante apresentações de seminários ou discussões sobre o tema em pauta. A problemática da conectividade no Brasil é ainda um desafio a ser superado. Segundo De Barros e De Oliveira ${ }^{10}$, apesar da implementação do Programa Nacional de Banda Larga em 2010, o Brasil ainda não alcançou êxito no que diz respeito ao acesso, com maior cobertura, bem como qualidade do serviço.

Outro ponto relevante refere-se ao fato de que muitos alunos não se conheciam, nem conheciam os docentes, nem mesmo o clima 
organizacional da instituição, o que representou mais uma dificuldade a ser superada à distância. A grande maioria dos alunos apresentava domínio de ferramentas digitais e internet, apesar disso não houve nenhuma ação de treinamento voltada para os discentes, depositando na esfera individual a responsabilidade pelo desenvolvimento dessas habilidades.

A virtualização da sala de aula, com sua inserção no domicílio e em outros locais, trouxe desdobramentos distintos. Apesar da comodidade de estar em casa, houve momentos em que a aula síncrona teve concorrentes, como afazeres domésticos, emergências pessoais e familiares e distrações (pessoas, televisão, celular entre outros).

Destaca-se, também, que a atividade profissional de alguns discentes foi convertida em trabalho remoto (home office), além da realização de cursos, reuniões de grupos de pesquisa e participação em eventos científicos, que assumiram o caráter online. Dessa forma, percebeu-se um aumento significativo do tempo de tela somando as aulas com outras atividades laborais, de estudo ou de lazer, que envolvem uso de aparelhos com tela (celular, TV, computador, tablets dentre outros).

O aumento no tempo despendido em atividades em que o gasto energético não é superior ao nível de repouso foi averiguado por Malta et al. ${ }^{11}$, no inquérito de saúde virtual "ConVid, Pesquisa de Comportamentos", realizado pelo Instituto Oswaldo Cruz em parceria com a Universidade Federal de Minas Gerais e a Universidade Estadual de Campinas. Observou-se que os entrevistados apresentaram aumento no tempo médio dedicado a assistir televisão (1 hora e 45 minutos), e a utilização de computador ou tablet aumentou, principalmente entre os adultos jovens (18-29 anos). Neste grupo etário, o aumento no tempo despendido foi na ordem de 3 horas, em relação ao verificado antes da pandemia.

Além disso, a necessidade do distanciamento social, como medida de prevenção e cuidado com a saúde individual e coletiva, impactou diretamente no nível de atividade física da população brasileira. Segundo os resultados do estudo de Malta et al. ${ }^{11}$, antes da pandemia, $30,1 \%$ dos entrevistados eram fisicamente ativos, porém, como consequência do isolamento, apenas $12 \%$ dos participantes conseguiram manter um nível suficiente de atividade física. Estas alterações no padrão comportamental, exemplificadas pelo aumento do comportamento sedentário e diminuição no nível de atividade física, mostra-se como uma realidade extremamente adversa, contribuindo para o aumento do risco de doenças metabólicas e cardiovasculares. ${ }^{12,13}$

Não obstante, ter que trabalhar e assistir aulas por meio de telas resultou na ocorrência de fadiga visual, dores de cabeça e dores musculares/articulares. Possivelmente, tais aspectos sejam resultantes da realização das atividades acadêmicas e de trabalho em casa, em condições ergonômicas desfavoráveis, já que para realização destas demandas no formato presencial antecedente à pandemia, contava-se com as instalações da universidade, que possui salas de aula, núcleos de pesquisas e a biblioteca. Ou seja, o ambiente destinado ao ensino remoto pode ser desconfortável e menos propício para tais atividades, como observado por Dosea et al. ${ }^{14}$, onde o local de estudo domiciliar foi apontado como uma das principais dificuldades de universitários nesta modalidade de ensino.

Um ponto positivo, percebido especialmente pelos discentes que residem em outras cidades, foi a economia com transporte, hospedagem e alimentação, o que gerou uma maior comodidade. A otimização do tempo com a redução dos deslocamentos permitiu melhor gerenciamento do tempo para realização das atividades do doutorado.

Neste contexto, esta modalidade de ensino pode ser considerada uma possível estratégia a ser adotada para locais distantes dos grandes centros urbanos, onde a oferta de vagas em programas de pós-graduação seja pequena ou inexistente. Ressalta-se que, segundo o Plano Nacional de Pós-Graduação 2011-2020, a região Sudeste concentra a oferta de programas de pósgraduação no país, sendo a região Norte a que possui o menor número. ${ }^{15}$

Entretanto, apesar de reconhecer esta potencialidade, o ensino remoto na pósgraduação ainda é um imenso desafio, uma vez que, não basta disponibilizar as aulas e professores. O Brasil é um país com elevada iniquidade social e, de forma geral, a população que possivelmente seria a mais beneficiada com esta modalidade de ensino, é a que, talvez, possua menores condições para se adequar às peculiaridades que este formato de ensino exige. Saraiva et al. $^{16}$ deixam clara a existência das desigualdades de condições observadas na modalidade de ensino remoto, ao comparar sua prática no ensino público e privado, não se resumindo apenas ao acesso à internet, mas também, ao contexto social.

Além disso, apesar da boa relação online entre os envolvidos, a ausência de contato físico 
e de comunicação presencial foi desafiador. Momentos de convívio e de interação, que geram aproximações e diálogos, enriquecendo as relações interpessoais e a construção de laços, fizeram falta no cotidiano dos estudantes. Segundo Valente et al. ${ }^{17}$, o processo de ensinoaprendizagem na modalidade presencial não pode ser substituído, no que diz respeito à convivência social proporcionada no ambiente universitário, destacando ainda a necessidade de uma construção coletiva de novas habilidades e saberes que tornem o processo mais dinâmico na modalidade online.

\section{Considerações finais}

De forma geral, não foram percebidas grandes dificuldades por parte dos discentes. A adaptação à modalidade de aulas remotas nas disciplinas do doutorado foi satisfatória. Houve boa adesão dos discentes e um esforço conjunto dos alunos e professores para que a comunicação fosse efetiva, por meio de e-mail e grupos de WhatsApp.

Diante da experiência relatada, percebese grande potencial da modalidade remota em substituir algumas atividades da pós-graduação, como reuniões de grupos de trabalho, aulas onde a atividade não envolver uma participação síncrona do discente (assistir a documentários/vídeos, leitura e produção de textos/resenhas, dentre outros), aulas com professores convidados que residem em outras cidades etc.

As dificuldades apresentadas com o acesso à internet, que contaram com a compreensão dos docentes, ilustram um dos grandes desafios do ensino remoto no Brasil. Destaca-se, então, a necessidade de expansão das políticas públicas que garantam o acesso à internet e a ferramentas digitais adequadas, contribuindo para o êxito do ensino remoto.

Não é possível anular o efeito negativo da pandemia da COVID-19 na qualidade de vida e desempenho dos atores envolvidos. Discentes, docentes e seus entes queridos viram-se isolados, com medo, alguns acometidos pela doença, o que pode ter impactado na dinâmica familiar e social, saúde mental e estabilidade emocional dos mesmos.

\section{Referências}

1. Organização Pan-Americana de Saúde/Organização Mundial de Saúde - Folha informativa - COVID-19 (doença causada pelo novo coronavírus), 2020. [Citado 2020 nov 24]. Disponível em: https://www.paho.org/pt/covid19

2. Aquino EML, Silveira IH, Pescarini JM, Aquino $R$, Souza-Filho JA, Rocha $A L$, et al. Medidas de distanciamento social no controle da pandemia de COVID-19: potenciais impactos e desafios no Brasil. Cien Saude Colet. 2020;25(Supl.1):2423-2446.

3. Brasil. Portaria no 343, de 17 de março de 2020. Dispõe sobre a substituição das aulas presenciais por aulas em meios digitais enquanto durar a situação de pandemia do Novo Coronavírus - COVID-19. Diário Oficial da União. Brasília, Distrito Federal, 2020 [Citado 2020 nov 24]. Disponível em: http://www.in.gov.br/web/dou/-/portaria-n-343de-17-de-marco-de-2020-248564376

4. Vercelli LCA. Aulas remotas em tempos de covid-19: a percepção de discentes de um programa de mestrado profissional em educação. Revista@@mbienteeducação. 020;13(2):47-60.

5. Bezerra KP, Costa KPL, Oliveira LC, Fernandes ACL, Carvalho FPB, Nelson I, et al. Ensino remoto em universidades públicas estaduais: o futuro que se faz presente. Res., Soc. Dev. 2020;9(9):e359997226.

6. World Health Organization. WHO Director-General's opening remarks at the media briefing on COVID-19 - 11 March 2020. World Health Organization, 2020. [Citado 2020 nov 24]. Disponível em: https://www.who.int/dg/speeches/detail/whodirector-general-s-opening-remarks-at-themedia-briefing-on-covid-19---11-march-2020/ 7 Brasil. Portaria no 544, de 16 de junho de 2020. Dispõe sobre a substituição das aulas presenciais por aulas em meios digitais, enquanto durar a situação de pandemia do novo coronavírus - Covid-19, e revoga as Portarias MEC no 343, de 17 de março de 2020, no 345, de 19 de março de 2020, e no 473, de 12 de maio de 2020. Diário Oficial da União. Brasília, Distrito Federal, 2020. [Citado 2020 nov 24]. Disponível em: http://www.in.gov.br/en/web/dou/-/portaria-n544-de-16-de-junho-de-2020-26192487

8. Universidade Estadual do Sudoeste da Bahia. Conselho Superior de Pesquisa e ExtensãoCONSEPE. Resolução CONSEPE № 22/2020. Regulamenta, em caráter excepcional, a adoção de atividades pedagógicas não presenciais (Ensino Remoto Emergencial-ERE), no ensino de graduação da UESB, durante o período da pandemia. Vitória da Conquista: CONSEPE, 2020. [Citado 2021 jan 05]. Disponível em: http://www.uesb.br/evidencias/resolucaoconsep 
e-022-2020-regulamento-do-ensino-remotoemergencial-para-os-cursos-de-graduacao/ 9. Universidade Estadual do Sudoeste da Bahia. Conselho Superior de Pesquisa e ExtensãoCONSEPE. Resolução CONSEPE № 23/2020. Regulamenta a oferta de componentes curriculares no formato de Ensino Remoto Emergencial (ERE) e estabelece condições para o desenvolvimento de outras atividades acadêmicas da pós-graduação stricto sensu da UESB, no contexto de suspensão das atividades acadêmicas presenciais em decorrência da pandemia da Covid-19. Vitória da Conquista: CONSEPE, 2020. [Citado 2020 nov 24]. Disponível em: http://www.uesb.br/evidencias/resolucaoconsepe-023-2020-regulamento-do-ensinoremoto-emergencial-na-pos-graduacao/

10. De Barros BMC, De Oliveira RS. A inclusão digital e o acesso à internet no Brasil sob a perspectiva dos avanços e retrocessos do programa nacional de banda larga-pnbl. Revista Paradigma. 2017;26(1):213-230.

11. Malta DC, Szwarcwald CL, Barros MBA, Gomes CS, Machado IE, De Souza Júnior PRB, et al. A pandemia da COVID-19 e as mudanças no estilo de vida dos brasileiros adultos: um estudo transversal, 2020. Epidemiol Serv Saude. 2020; 29:2020407.

12. Meneguci J, Santos DAT, Silva RB, Santos RG, Sasaki JE, Tribess $S$, et al. Comportamento sedentário: conceito, implicações fisiológicas e os procedimentos de avaliação. Motricidade. 2015;11(1):160-174.

13. Mainous AG, Tanner RJ, Rahmanian KP, Jo A, Carek PJ. Effect of sedentary lifestyle on cardiovascular disease risk among healthy adults with body mass indexes 18.5 to $29.9 \mathrm{~kg} / \mathrm{m}^{2}$. Am J Cardiol. 2019;123(5):764-768.

14. Dosea GS, Silva EA, Oliveira AMS, Rosário RWS, Firmino LR. Métodos ativos de aprendizagem no ensino online: a opinião de universitários durante a pandemia de covid19. EDU [Internet]. 2020;10(1):137-148.

15. Brasil. Ministério da Educação. Coordenação de Aperfeiçoamento de Pessoal de Nível Superior (CAPES). Plano Nacional de PósGraduação: PNPG 2011-2020. Brasília-DF: CAPES, 2010.

16. Saraiva K, Traversini C, Lockmann K. A educação em tempos de COVID-19: ensino remoto e exaustão docente. Práxis Educativa. 2020; 15: e2016289,1-24.

17. Valente GSC, Moraes EB, Sanchez MCO, Souza DF. O ensino remoto frente às exigências do contexto de pandemia: reflexões sobre a prática docente. Res., Soc. Dev. 2020;9(9):e843998153.

\section{Endereço para Correspondência}

Thalane Souza Santos Silva

Av. José Moreira Sobrinho, s/n - Jequiezinho, Jequié, Bahia, Brasil. CEP 45205-490

E-mail: thalanesouza@hotmail.com

Recebido em 07/04/2021

Aprovado em 22/05/2021

Publicado em 27/09/2021 